\title{
Study protocol to assess the effectiveness of pharmacist- managed stop-smoking services : A randomized controlled trial
}

\author{
Sam J. U. Chander ${ }^{1}$, Subramanian Santhakumar ${ }^{2}$, Sam W. D. Solomon ${ }^{3}$
}

\author{
AFFILIATION \\ 1 Department of Pharmacology, College of Pharmacy, Sri Ramakrishna \\ Institute of Paramedical Sciences, Tamil Nadu, India \\ 2 KG Hospital and Postgraduate Medical Institute, Tamil Nadu, India \\ 3 Department of Pharmaceutical Analysis, RVS College of Pharmaceutical \\ Sciences, Tamil Nadu, India
}

\section{CORRESPONDENCE TO}

Sam Johnson Udaya Chander J. Department of Pharmacology, College of Pharmacy, Sri Ramakrishna Institute of Paramedical Sciences, Coimbatore 641044, Tamil Nadu, India. E-mail: mail2samjohnson@gmail.com

\section{KEYWORDS}

smoking cessation, pharmacist, India, nicotine dependence

Received: 4 January 2020, Revised: 17 February 2020,

Accepted: 17 February 2020

\begin{abstract}
According to the Global Adult Tobacco Survey (GATS), there were 267 million tobacco users in India during 2016-2017. It is also estimated that about one million people die each year due to tobacco-related diseases in India. Tobacco cessation is the only way by which tobacco-related morbidity and mortality can be avoided. Healthcare workers are ideal professionals to encourage smoking cessation, and pharmacists are the most accessible healthcare providers who can initiate a change in the smoking behavior of patients. Many studies, worldwide, have demonstrated the effectiveness of pharmacists in delivering a smoking cessation program, but studies demonstrating the effectiveness of pharmacist-managed smoking cessation services in India are almost non-existent. This protocol aims to test the effectiveness of Pharmacist-Managed Stop Smoking Services $\left(\mathrm{PM}-\mathrm{S}^{3}\right)$, a structured smoking cessation program delivered by a clinical pharmacist.
\end{abstract}

A prospective, randomized, controlled trial is being conducted at a 550-bed tertiary care hospital in Coimbatore, India. Participants are randomly assigned to either an interventional group, where they receive at least four sessions of face-to-face, patient-specific, structured smoking cessation counselling by a clinical pharmacist for approximately 30 minutes each, or to usual-care group where they receive brief smoking cessation counseling by a physician and/or nurse. Both groups are provided with nicotine replacement therapy, if warranted. The primary outcome of smoking cessation is measured in two ways, viz self-reported abstinence and an exhaled carbon monoxide (CO) test.

If proven effective, this model can be used as a framework for establishing pharmacist-managed stop smoking clinics in all hospitals throughout India to decrease the overall smoking burden.

\section{INTRODUCTION}

Tobacco use is the leading cause of preventable death worldwide ${ }^{1}$. Approximately 6 million premature deaths related to tobacco use occur globally each year, of which approximately 0.6 million are related to secondhand smoke ${ }^{2}$. According to the Global Adult Tobacco Survey-India, there were 267 million tobacco users (37.5\% were smokers) in 2017 in India, second only to China ${ }^{3}$. Tobacco use remains a major preventable cause of Chronic Obstructive Pulmonary Disease (COPD) and lung cancer ${ }^{4}$. COPD is a significant cause of morbidity and mortality worldwide. COPD kills more than 3 million people globally, making it the fourth most significant cause of death and it is estimated that by 2030 , COPD will become the third largest cause of preventable death worldwide ${ }^{5}$. In India, COPD is recognized as a major health condition that requires care from various levels of healthcare professionals 6 , while tobacco smoking is responsible for over $82 \%$ of COPD cases in India ${ }^{7,8}$. Since smoking is the primary risk factor for COPD, smoking cessation is the key to reduce the prevalence of COPD, and 
the morbidity and mortality associated with COPD. Smoking cessation also improves the quality of life and confers considerable health benefits ${ }^{9}$. Smoking cessation can be achieved through motivational counselling by a healthcare professional and is well supported by the literature as an effective strategy to bring about a positive outcome among smokers $^{10}$. Until 2002, there were no formal smoking cessation services available in India and the first smoking cessation clinic was set up in 2002 as a joint initiative of the Ministry of Health and Family Welfare, the Government of India and the WHO. Later, many such clinics were developed to minimize the burden of tobacco smoking in India ${ }^{11}$. Due to limited time for and direct access of physicians to patients, especially in a country like India, utilizing the services of nondoctor healthcare professionals who can administer smoking cessation counselling more frequently than physicians is warranted $^{12}$. A study done in Kerala, India, showed that smoking cessation intervention done by a non-doctor healthcare professional increased the quit rate by $40 \%$ in the intervention group compared to the control group at six months follow-up ${ }^{13}$. Among healthcare professionals, pharmacists may be in a better position to provide smoking cessation counselling, as they have more opportunity for face-to-face interaction with patients.

Moreover, many pharmacies are open until late evening and some are open continuously for 24 hours $^{14}$. Since clinical pharmacy education is a decade old in India, clinical pharmacists may be in a key position to provide smoking cessation services in India due to their proximity to patients and the level of interaction they have with patients in the clinical practice ${ }^{15,16}$. Different studies conducted in countries such as the USA, Canada, Indonesia, Thailand, Poland and Sudan, report the significant role of the pharmacist in implementing smoking cessation services, but studies demonstrating the effectiveness of pharmacist-led smoking cessation services in India are non-existent.

\section{Study objectives}

This study protocol describes a program that is currently being implemented in a 550-bed tertiary care hospital in Coimbatore, India. The study hypothesis is that structured, patient-specific, smoking cessation counselling delivered by a clinical pharmacist will be more effective in motivating smokers to stop smoking and achieving long-term abstinence from smoking cigarettes. Hence, the objective of this study was to compare the effectiveness of a structured, patientspecific, smoking cessation intervention by a clinical pharmacist with the intervention provided by other healthcare professionals in a clinic setting in India.

\section{METHODS}

This study follows the SPIRIT 2013 statement, which provides recommendations for a minimum set of scientific, ethical and administrative elements that should be addressed in a clinical trial protocol ${ }^{17}$.

\section{Study design}

This study is a prospective, open-label, randomized controlled trial studying the effectiveness of a face-to-face structured smoking cessation program conducted by a clinical pharmacist. The study protocol is named Pharmacist-Managed Stop Smoking Services (PM-S ${ }^{3}$ ), a novel program implemented to promote pharmacists within the profession as effective providers of smoking cessation counseling and help to patients to become smoking-free and have healthier lives, in India.

\section{Study setting}

The PM-S ${ }^{3}$ is implemented in the Pulmonology Department of a 550-bed tertiary care hospital in Coimbatore, India.

\section{Study pharmacist's training}

Prior to starting the study, the study methodology was discussed with a panel of pulmonologists and general physicians in four tertiary care hospitals in Coimbatore, India. With the help of the panel, the study pharmacist designed the strategy for smoking cessation counselling and also a booklet to be distributed to each participant.

\section{Study marketing}

Since this is a new intervention, the study pharmacist had to effectively market the PM-S ${ }^{3}$ Program. The study pharmacist displayed posters and leaflets describing the smoking cessation program in the pulmonology clinic of the study hospital. A letter of invitation was also sent to other pulmonology clinics in the Coimbatore City, asking them to refer their patients to the $\mathrm{PM}-\mathrm{S}^{3}$ clinic.

\section{Screening for eligibility}

The willingness of patients to quit smoking is checked by a physician before starting the study. Smokers wishing to quit will be referred to the PM-S ${ }^{3}$ Program and the clinical pharmacist will administer a structured data collection form specially prepared for this study in order to assess their eligibility to be included in the study. Sociodemographic information such as name, age, sex, smoking history, past medical history, family history, allergic history, duration of the disease, symptoms, diagnosis, educational background, and address will be collected. Eligible participants are: patients aged $\geq 18$ years; diagnosed with COP; currently smoke one or more cigarettes daily; willing to quit smoking tobacco; able to communicate in either English or Tamil; and willing and capable of attending all the scheduled sessions in the PM-S ${ }^{3}$ program. Exclusion criteria include: planning to leave India in the next 12 months; use of other tobacco products; use of smoking cessation aids currently or within the last 30 days; being pregnant; and having a psychiatric condition or other major medical issues that would not allow the patient to participate in the program.

\section{Study enrollment}

After the completion of the eligibility screening procedure, 
the participants will be informed by the study pharmacist about the $\mathrm{PM}-\mathrm{S}^{3}$ program, the potential benefits, any inconvenience and discomfort, risks, and the right to confidentiality. As a prerequisite of enrollment, each participant will be asked to sign an informed consent form, printed in both English and Tamil. After signing the informed consent form, the study pharmacist will collect the participant's contact information and a mutually convenient date and time will be set for an initial appointment.

\section{Initial appointment and randomization}

One to two days before the scheduled appointment, the study pharmacist will contact the enrolled participants and explain the initial appointment. The initial appointment will take about half an hour during which the study pharmacist will first collect the sociodemographic data, present medical problems and medications, obtain smoking history (number of cigarettes per day, duration of smoking, previous quit attempts, and participant's reason for quitting smoking), and vital signs. After this, the study pharmacist will assess the nicotine dependence of the participants using the Fagerström Test for Nicotine Dependence. All participants will be categorized into one of the five 'stages of readiness to change'18. These are: 1) Pre-contemplation - no intention to quit smoking; 2) Contemplation - aware that smoking is injurious, but is ambivalent about quitting and hence no quit date is planned; 3) Preparation - with an intent to quit in six months; 4) Action - with an intent to quit in one month or has already quit in the last month; and 5) Maintenance - has quit for more than one month. After baseline data collection, the patients will be placed into either the control group or interventional group using a simple randomization method where odd listed patients become the control group and even listed patients the intervention group. A new appointment will then be set for participants assigned to the intervention group. The participants in the control group will receive brief unstructured smoking cessation counselling whereas participants in the intervention group will receive additional care and structured smoking cessation advice by the study pharmacist, based on the five significant steps (the '5 As') provided by the US Public Health Service Clinical Practice Guidelines ${ }^{19}$. When patients are asked: 'How sure are you to make a quit attempt at this time?', they are expected to rate themselves on a motivation scale of $0-10$, with 10 most motivated to quit. If patients are not sure about quitting, they are rated between 1 and 3 , and are considered to be in the pre-contemplation stage. The patients in this stage have to move to the contemplation stage in order to become eligible for the intervention. If patients score 4 or 5 , they are considered to be in the contemplative stage and unsure of quitting. When the patients score $\geq 6$, they are somewhat sure or extremely sure of quitting and become prime candidates for smoking cessation counselling. To enhance motivation, the patients in the pre-contemplation and contemplation stages benefit most from one-on-one counselling using 'the $5 \mathrm{Rs}^{\prime 19}$. Once patients move from pre-contemplation to contemplation, they are most likely to succeed in smoking cessation counselling. Participants of both the intervention and usual-care groups do not pay any fee for their participation.

\section{Intervention group}

Participants assigned to the intervention group will receive four sessions of a face-to-face program at the smoking cessation clinic, delivered by the study pharmacist, over one year. The appointments are set at a date and time convenient to both participant and study pharmacist.

\section{First session}

This is time-consuming, taking sometimes more than one hour. In this session, the study pharmacist facilitates the participant's preparation to quit. The study pharmacist aims to advance the participants in the contemplation stage to the preparation stage. The participants select a quit date within the next three to four weeks. Based on the transtheoretical model of change, this would give the participants time to get prepared to enter the action stage on the set quit date. During this session, the study pharmacist discusses the benefits of quitting smoking with the participants and provides them with suggestions for appropriate behavioral and lifestyle changes. The study pharmacist also provides the participants with information on what to expect during the early days of quitting. The participants will also be encouraged to seek family support to help their quit attempt. If necessary, the participants will be provided with nicotine replacement therapy (NRT) in the form of a patch or a lozenge by a consultant pulmonologist to prevent nicotine withdrawal symptoms. Participants, who take the nicotine patch, start at $21 \mathrm{mg}$ if they smoke ten or more cigarettes per day. Otherwise, they receive a 14-mg patch. Both doses are continued for six weeks before being tapered off. The 21-mg patch is decreased to $14 \mathrm{mg}$, continued for two weeks and then decreased to $7 \mathrm{mg}$ for the final two weeks. Patients who start with $14 \mathrm{mg}$ are tapered off to $7 \mathrm{mg}$ for the final two weeks ${ }^{20}$. Participants who take the nicotine lozenge use the 1 -mg pieces. This is continued for six weeks with one lozenge every one to two hours before being tapered off. The patient takes one lozenge every two to four hours for weeks 7-9, then one lozenge every four to eight hours for weeks $10-12^{21}$. A personalized action plan is created by the study pharmacist to document the participant's quit date, nicotine replacement therapy regimen, behavioral and lifestyle strategies, and date and time of next appointment. This plan is jointly signed by the participant, the study pharmacist and the consultant pulmonologist.

\section{Follow-up sessions}

The first follow-up session is scheduled two weeks after the participant's quit date and takes approximately 30 minutes. In this session, the study pharmacist determines 
the participant's smoking status, measures blood pressure, pulse, exhaled CO level and assesses their tolerability to NRT. If the participant fails the quit attempt, the study pharmacist carefully reviews the participant's experience and identifies the problems and works through the identified problems. If the participant succeeds in the quit attempt, the study pharmacist reinforces the support provided and addresses the problems associated with nicotine withdrawal, relapse issues, relationship issues with friends and society, and cravings. If the participant does not tolerate nicotine replacement therapy, he/she will be referred back to the consultant pulmonologist and stopped from continuing in the study. The second follow-up session is scheduled four weeks after the first follow-up session, and the third followup session is scheduled six weeks after the second follow-up session. During these visits, the study pharmacist supports the patients to remain abstinent and prevent relapses. The study pharmacist documents any action taken by the patients at each follow-up session. After the third follow-up visit, all patients will be instructed to visit the clinic for clarification or to receive additional support.

\section{Control group}

The participants in the usual-care group will receive usual hospital outpatient care from medical and nursing staff, but will not receive the structured, patient-specific smoking cessation intervention referred to above.

\section{Educational material}

The educational material will cover the following topics: ill effects of smoking, benefits of smoking cessation, methods to quit, setting the quit date, coping with nicotine withdrawal symptoms, getting emotional and social support, how to use NRT, and avoid relapse.

\section{Study piloting}

The study pharmacist was assisted to set up the PM-S ${ }^{3}$ Program and given time to familiarize with the study protocol before the commencement of the study. The study was piloted by recruiting about six patients and implementing the study protocol. Feedback was provided on the quality of patient counselling and patient education. Minor adjustments were made to the consent form, data collection form, and smoking progress note as indicated by the panel of pulmonologists and general physicians.

\section{Continuous quality improvement and evaluation}

The study pharmacist receives constructive feedback from the panel of pulmonologists and general physicians. In addition, an ad hoc meeting will be held to address any irregular issues that might arise during the study.

\section{Outcome measures}

The primary outcome measure is smoking abstinence. This will be measured in two ways. First, self-reported abstinence during the past one week will be recorded at each review meeting. The patient who misses the session will be considered a smoker at that point. Second, an exhaled carbon monoxide (CO) test (BreathCO) will be administered at each session completion. Any participant who does not perform the CO test is considered a smoker at that point in time. The outcomes will be measured at each follow-up visit.

\section{Statistical analyses}

Sociodemographics and other variables related to smoking will be summarized using frequency distribution for categorical variables and means with standard deviation for numerical variables. The effectiveness of the program will be established by chemically verified smoking abstinence and this number will be the participants who achieved smoking abstinence at the end of the program. Baseline differences between quitters and non-quitters will be analyzed by Fisher's exact test or independent sample t-tests. The primary outcome will be computed and compared between the two groups using the chi-squared test.

\section{Sample size calculation}

The recruitment period is 12 months. Around 40 COPD patients visit the pulmonology clinic each day out of which approximately 25 are males of whom approximately 10 are smokers. Expecting that the study will recruit 5 to 6 patients every week, a minimum sample size of 100 participants was estimated for each group. An extra 8 weeks of recruitment time will be considered to account for holidays and sickness.

\section{DISCUSSION}

This study is likely to be the first randomized controlled trial conducted within India to compare the effectiveness of structured, patient-specific, smoking cessation intervention by a clinical pharmacist with the intervention provided by physicians and/or nursing staff in a clinic setting in India. This study will be of significance to India and to some extent for South Asia. This study will shed light on the additional role of clinical pharmacists in India and will clearly show to what extent a clinical pharmacist can be useful as a provider of smoking cessation intervention. Previous studies, conducted in USA, Europe and Australia, have demonstrated the effectiveness of a pharmacist-run smoking cessation program ${ }^{22-29}$, but merely extrapolating their results to India is not valid as the pharmacy practice in India is at its infancy and greatly differs from that of the these countries. Pharmacists in India are interested in an additional new welldefined vocation for themselves by including in their focus interventions for patients.

\section{CONCLUSIONS}

The outcomes of this study will reposition the clinical pharmacists in India within a broader perspective as healthcare providers than merely being medicine dispensers. By involving clinical pharmacists in smoking cessation 
counselling, the professional role of pharmacists will be upgraded to include smoking cessation motivation with a more patient-centered focus. This will help the clinical pharmacists to better integrate within the healthcare teams in India.

\section{REFERENCES}

1. World Health Organization. WHO report on the global tobacco epidemic, 2013 -Enforcing bans on tobacco advertising, promotion and sponsorship. Geneva, Switzerland: World Health Organization; 2013. http:// www.who.int/tobacco/global_report/2013/en/index.html. Accessed January 4, 2020.

2. Oberg M, Jaakkola MS, Woodward A, Peruga A, PrüssUstün A. Worldwide burden of disease from exposure to second-hand smoke: A retrospective analysis of data from 192 countries. Lancet. 2011;377(9760):139-146. doi:10.1016/s0140-6736(10)61388-8

3. World Health Organization. GATS 2: Global Adult Tobacco Survey Fact Sheet, India 2016-17. https://www.who.int/ tobacco/surveillance/survey/gats/GATS_India_2016-17_ FactSheet.pdf?ua=1. Accessed Jnauary 4, 2020.

4. Brose LS, West R, Mcdermott MS, et al. What makes for an effective stop-smoking service? Thorax. 2011;66(10):924926. doi:10.1136/thoraxjnl-2011-200251

5. Sundeep Salvi, Aurag Agarwal. India Needs a National COPD Prevention and Control Programme. J Assoc Physicians India. 2012;60(Suppl):5-7. PMID:23155805.

6. Jindal SK, Gupta D, Aggarwal AN. Guidelines for management of chronic obstructive pulmonary disease in India: a guide for physicians. Indian J Chest Dis Allied Sci. 2004;46(2):137-193. PMID:15072332.

7. Jindal SK, Aggarwal AN, Gupta D. A review of population studies from India to estimate national burden of chronic obstructive pulmonary disease and its association with smoking. Indian J Chest Dis Allied Sci. 2001;43(3):139-147. PMID:11529432.

8. World Health Organization. Report on Tobacco Control in India. New Delhi, India: Ministry of Health and Family Welfare, Government of India; 2004. https://www.who. int/fctc/reporting/Annex6_Report_on_Tobacco_Control_in_ India_2004.pdf. Accessed January 4, 2020.

9. Centers for Disease Control and Prevention: Smoking and Tobacco Use Cessation and Interventions. http://www.cdc. gov/tobacco/data_statistics/fact_sheets/cessation/quitting/ index.htm. Accessed Januaru 4, 2020.

10. Roth MT, Andrus MR, Westman EC. Outcomes from an outpatient smoking cessation clinic. Pharmacotherapy. 2005;25(2):279-288. doi:10.1592/phco.25.2.279.56957

11. Raj Kumar, Rajendra Prasad. Smoking Cessation: An Update. Indian J Chest Dis Allied Sci. 2014;56(3):161-169. PMID:25823111.

12. Rao M, Rao KD, Kumar AK, Chatterjee M, Sundararaman T. Human resources for health in India. Lancet. 2011;377(9765):587-598. doi:10.1016/s0140-6736(10)61888-0
13. Thankappan KR, Mini GK, Hariharan M, Sarma PS, Vijayakumar G, Nichter M. Smoking cessation among diabetes patients: one year follow-up results of a randomized controlled trial in Kerala, India. Diabetes. 2013;13(1):A1A98. doi:10.1186/1471-2458-13-47

14.Vitale F. Professional intervention for smoking cessation. The contribution of the pharmacist. Eur J Public Health. 2000;10(3):21-24. doi:10.1093/eurpub/10.suppl_3.21

15. Dhingra S, Kumria R. A case report on the significance of clinical pharmacy services in India. Clin Case Rep. 2014;2(3):86-87. doi:10.1002/ccr3.63

16. Dent LA, Harris KJ, Noonan CW. Randomized trial assessing the effectiveness of a pharmacist-delivered program for smoking cessation. Ann Pharmacother. 2009;43(2):194-201. doi:10.1345/aph.11556

17. Chan AW, Tetzlaff JM, Altman DG, et al. SPIRIT 2013 Statement: Defining Standard Protocol Items for Clinical Trials. Ann Intern Med. 2013;158(3):200. doi:10.7326/0003-4819-158-3-201302050-00583

18. Hudmon KS, Berger BA. Pharmacy applications of the transtheoretical model in smoking cessation. Am J Health Syst Pharm. 1995;52(3):282-287. doi:10.1093/ajhp/52.3.282

19. Fiore MC, Bailey WC, Cohen SJ, et al. Treating tobacco use and dependence. Clinical practice guideline. Rockville, MD: U.S. Department of Health and Human Services, Public Health Service; 2000.

20.DRUGDEX ${ }^{\circledR}$ Evaluations: Nicotine. http://www. micromedexsolutions.com/home/dispatch. Accessed January 4, 2020.

21.DRUGDEX® Evaluations: Nicotine polacrilex. http://www. micromedexsolutions.com/home/dispatch. Accessed January 4, 2020.

22. Maguire TA, McElnay JC, Drummond A. A randomized controlled trial of a smoking cessation intervention based in community pharmacies. Addiction. 2001;96(2):325-331. doi:10.1046/j.1360-0443.2001.96232516.x

23. Vial RJ, Jones TE, Ruffin RE, Gilbert AL. Smoking cessation program using nicotine patches: linking hospital to the community. J Pharm Pract Res. 2002;32(1):57-62. doi:10.1002/jppr200232157

24. Carroll P, Rois R, Sarson D, Flynn J. A community pharmacy based smoking cessation program using transdermal nicotine replacement therapy: actions and outcomes. Aust Pharmacist. 2000;19:51-55.

25. Sinclair HK, Bond CM, Lennox AS, Silcock J, Winfield AJ, Donnan PT. Training pharmacists and pharmacy assistants in the stage-of-change model of smoking cessation: a randomized controlled trial in Scotland. Tob Control. 1998;7(3):253-261. doi:10.1136/tc.7.3.253

26.Zillich AJ, Ryan M, Adams A, Yeager B, Farris K. Effectiveness of a pharmacist-based smoking-cessation program and its impact on quality of life. Pharmacotherapy. 2002;22(6):759765. doi:10.1592/phco.22.9.759.34073

27. Smith MD, McGhan WF, Lauger G. Pharmacist counseling and outcomes of smoking cessation. Am Pharm. 
1995;NS35(8):20-29. doi:10.1016/s0160-3450(15)30095-7

28. Kennedy DT, Giles JT, Chang ZG, Small RE, Edwards JH. Results of a smoking cessation clinic in community pharmacy practice. J Am Pharm Assoc. 2002;42(1):51-56. doi:10.1331/108658002763538071

29. Dent LA, Scott JG, Lewis E. Pharmacist-managed tobacco cessation program in Veterans Health Administration community-based outpatient clinic. J Am Pharm Assoc. 2004;44(6):700-715. doi:10.1331/1544345042467290

\section{ACKNOWLEDGEMENTS}

We are grateful to the study community and hospital authorities and workers for their support during study implementation.

\section{CONFLICTS OF INTEREST}

The authors have completed and submitted the ICMJE Form for Disclosure of Potential Conflicts of Interest and none was reported.

FUNDING

There was no source of funding for this research.
AUTHORS' CONTRIBUTIONS

SJUC participated in conception of the study, study design, and drafted this protocol. SS and SSWD participated in study design. SJUC and SS will assess the effect of the intervention. All authors read and approved the final manuscript.

PROVENANCE AND PEER REVIEW

Not commissioned; externally peer reviewed. 